\title{
Controlling fungal contamination in Chlamydomonas reinhardtii cultures
}

\author{
Kristina M. Mahan, Obed W. Odom, and David L. Herrin \\ University of Texas at Austin, Austin, TX, USA
}

BioTechniques 39:457-458 (October 2005)

doi 10.2144/000112022

Chlamydomonas reinhardtii is a unicellular eukaryotic green alga that is used widely for the study of photosynthesis, organelle biogenesis, and cell motility, among other processes (1). $C$. reinhardtii is a model genetic organism that grows on solid (agar) or liquid media and can do so autotrophically (light), heterotrophically (dark plus acetate), or mixotrophically (light plus acetate). The ability of $C$. reinhardtii to grow heterotrophically on acetate has allowed investigators to isolate and maintain nonphotosynthetic mutants with relative ease. Mixotrophic growth conditions are probably the most common for culturing $C$. reinhardtii, because most strains grow more rapidly under these conditions than with the other trophic states (2). The acetatecontaining media, however, also supports vigorous growth of unwanted fungi, and with the slowly growing strains of $C$. reinhardtii, the cultures can be completely overrun and ruined by fungal contaminants. The fungal problem can be particularly severe in locations where there are high levels of airborne spores, such as in geographic areas with high humidity or in older buildings.

Current methods for controlling fungal contamination in $C$. reinhardtii rely on using aseptic technique when handling cultures (to prevent contamination) and, in the case of contaminated cultures, physical separation of algal cells from fungal cells (on agar plates) under a microscope (2). Also, some strains of $C$. reinhardtii show positive phototaxis, and this ability can be used to physically clean cultures, albeit liquid in this case. Autotrophic growth, for those strains that are photosynthetic, can be used to reduce fungal contamination, but, in our experience, frequently does not provide a completely clean culture. This is probably because $C$. reinhardtii releases reduced small carbon compounds and proteins into the media that contaminants can apparently utilize (3).

A potentially convenient and effective control measure would be to add a fungicide to solid media to either prevent fungal contamination or to clean up contaminated cultures. However, no fungicides compatible with the growth of $C$. reinhardtii have been identified to our knowledge. Hence, we investigated the effect of a number of fungicides on the growth of a wild-type strain of $C$. reinhardtii (CC-3269) on solid Tris-acetatephosphate agar (TAP-agar) medium at $\mathrm{pH}$ 7.0. The selection of some of these fungicides for testing was based on previous studies with other green algae and a higher plant (4-6). The fungicides were dissolved in dimethylformamide, ethanol, or water and then added to the medium after autoclaving. Carbendazim was not completely soluble, so the medium containing this drug was remixed immediately before pouring each plate. After inoculation with either $C$. reinhardtii or the fungal contaminants that we had isolated free of algal cells, the plates were incubated at $23^{\circ} \mathrm{C}$ under moderate fluorescent light (approximately 500 lux) for 5 days and then scored for growth visually.

Table 1 summarizes the results obtained with most of the compounds that were tried. Three of the fungicides, carbendazim (Sigma, St. Louis, MO, USA), thiophanate-methyl (Chem Service, West Chester, PA, USA), and benomyl (from H.-H. Kim, Korea Institute of Standards and Technology), did not inhibit the growth of $C$. reinhardtii at concentrations up to 40 $\mu \mathrm{g} / \mathrm{mL}$, which is well within the fungicidal range for these drugs. In addition to vigorous growth, there also was no discoloration of the algae that grew at $40 \mu \mathrm{g} / \mathrm{mL}$ with these three drugs. The other three fungicides that were evaluated in Table 1 were too inhibitory to the algae to be of much use.

The effect of the fungicides on growth of our common fungal contaminants was also evaluated. These contaminants were identified as fungi based on their morphology, sensitivity to fungicides, and insensitivity to antibiotics that target bacteria. Also, based on morphology, there appeared to be at least two different species, although they were not assigned taxonomically. Table 1 shows that carbendazim was effective at the lowest concentration used $(1 \mu \mathrm{g} / \mathrm{mL})$, whereas thiophanate-methyl and benomyl effectively blocked fungal growth at 20 $\mu \mathrm{g} / \mathrm{mL}$ (there was a similar response by both contaminants). These and the aforementioned results indicate that all three of these compounds could be used in controlling fungal contamination of C. reinhardtii cultures.

These fungicides, carbendazim, thiophanate-methyl, and benomyl, are considered broad-spectrum fungicides of the benzimidazole family. They bind to microtubules and interfere with cell division and transport (Reference 7 and references therein). Currently, we are using mainly carbendazim, which has the additional benefit of being inexpensive, to clean up contaminated cultures. This is accomplished by streaking the contaminated algae onto TAP-agar plates containing $40 \mu \mathrm{g} / \mathrm{mL}$ carbendazim, and after 5-7 days of growth, the algae are restreaked back onto TAP-agar. Heavily contaminated cultures may require growth on the carbendazim plate two to three times in succession before restreaking the algae back onto TAP-agar. We have successfully cleaned a number of strains this way. We are also using these fungicides as a preventative measure when performing certain techniques that are prone to contamination, such as biolistic transformation.

This fungicide-based approach to control fungal contaminants is relatively straightforward and could be easily employed by inexperienced 
Table 1. Effect of Fungicides on the Growth of Chlamydomonas and Fungal Contaminants on Agar Plates

\begin{tabular}{|c|c|c|c|c|c|c|c|c|c|}
\hline \multirow[b]{2}{*}{ Organism } & \multirow[b]{2}{*}{ Fungicide } & \multicolumn{8}{|c|}{$\begin{array}{c}\text { Concentration } \\
(\mu \mathrm{g} / \mathrm{mL})\end{array}$} \\
\hline & & 1 & 10 & 20 & 40 & 70 & 90 & 100 & 160 \\
\hline \multirow{4}{*}{$\begin{array}{l}\text { Chlamydomonas } \\
\text { reinhardtii }\end{array}$} & Thiophanate-methyl & +++ & +++ & +++ & +++ & ++ & ++ & ++ & + \\
\hline & Benomyl & +++ & +++ & +++ & +++ & +++ & +++ & ++ & + \\
\hline & Amphotericin B & - & - & - & - & ND & ND & ND & ND \\
\hline & Anisomycin & + & - & - & - & ND & ND & ND & ND \\
\hline \multirow[t]{3}{*}{ Fungal contaminants } & Carbendazim & - & - & - & - & - & - & - & - \\
\hline & PPM & - & - & - & - & - & - & - & - \\
\hline & Anisomycin & +++ & +++ & +++ & +++ & ND & ND & ND & ND \\
\hline
\end{tabular}

researchers. One caveat that should be mentioned is that we have tested the fungicides only on $C$. reinhardtii growing vegetatively, which is the most common growth mode. We have not determined if there are effects of these drugs on gametes or zygotes. However, our suspicion is that these fungicides will likely be compatible with these other stages of the life cycle, in part because $C$. reinhardtii has only two $\beta$-tubulin genes, and they code for the same protein (8). Nonetheless, additional tests are being planned to look at the effect, if any, of these fungicides on zygote germination, so that they may be incorporated into the plates used for tetrad dissections (2).

\section{ACKNOWLEDGMENTS}

This research was supported by grants from the Department of Energy (DE-FG03-02ER15352) and the Robert A. Welch Foundation (F-1164).

\section{COMPETING INTERESTS STATEMENT}

The authors declare no competing interests.

\section{REFERENCES}

1.Harris, E.H. 2001. Chlamydomonas as a model organism. Annu. Rev. Plant Physiol. Plant Mol. Biol. 52:363-406.

2. Harris, E.H. 1989. The Chlamydomonas Sourcebook. Academic Press, San Diego.

3. Parker, B. and H.C. Bold. 1961. Biotic relationships between soil algae and other microorganisms. Am. J. Bot. 48:185-197.

4. Hunter, E.O. and I. McVeigh. 1961. The effects of selected antibiotics on pure cultures of algae. Am. J. Bot. 48:179-185.

5.Ma, J., R. Zheng, L. Xu, and S. Wang. 2002. Differential sensitivity of two green algae, Scenedesmus obliqnus, and Chlorella pyrenoidosa, to 12 pesticides. Ecotoxicol. Environ. Saf. 52:57-61.

6. Paul, A.-L. and C. Semer. 2001. The fungicidal and phytotoxic properties of benomyl and PPM in supplemented agar media supporting transgenic Arabidopsis plants for a space shuttle flight experiment. Appl. Microbiol. Biotechnol. 55:480-485.
7. Park, S.Y., O.J. Jung, Y.R. Chung, and C.W. Lee. 1997. Isolation and characterization of a benomyl-resistant form of beta-tubulin-encoding gene from the phytopathogenic fungus Botryotinia fuckeliana. Mol. Cells 28:104109.

8. Youngblom, J., J.A. Schloss, and C. Silflow. 1984. The two beta-tubulin genes of Chlamydomonas reinhardtii code for identical proteins. Mol. Cell. Biol. 4:2686-2696.

Received 3 July 2005; accepted 21 July 2005.

Address correspondence to David L. Herrin, Section of Molecular Cell and Developmental Biology and Institute for Cellular and Molecular Biology, 1 University Station A6700, University of Texas at Austin, Austin, TX 78712, USA. e-mail:herrin@mail.utexas.edu

To purchase reprints

of this article, contact

Reprints@BioTechniques.com 